\title{
Efeito do uso de agentes clareadores com cálcio na microdureza do esmalte dental
}

\section{Effect of bleaching agents with calcium in dental enamel microhardness}

\author{
Andreza Gruber* \\ Luciane Colli** \\ Carla Muniz ${ }^{* * *}$ \\ Rodrigo Ottoni $i^{* * *}$ \\ Márcia Borba ${ }^{* * * *}$
}

\section{Resumo}

Objetivo: avaliar a influência do uso de agentes clareadores com cálcio na microdureza do esmalte dental. Materiais e método: o estudo é experimental, laboratorial e realizado com dentes bovinos. Oito coroas de incisivos bovinos foram seccionadas em quatro partes e divididas em quatro grupos, de acordo com o tipo de agente clareador $(n=8)$ : (Con) protocolo de consultório (peróxido de hidrogênio 35\% - HP 35\%); (Com+Ca) protocolo de consultório com cálcio (HP 35\%); (Cas) protocolo caseiro (peróxido de carbamida 10\%); (Cas+Ca) protocolo caseiro com cálcio (HP 6\%). As amostras foram tratadas de acordo com as recomendações do fabricante para cada agente clareador. A microdureza do esmalte foi avaliada antes, imediatamente após e 7 dias após os tratamentos clareadores. Foi utilizado um microdurômetro e ponta Vickers, com uma carga de 200 g por 15 segundos. As amostras permaneceram em água destilada a $37^{\circ} \mathrm{C}$ durante todo o experimento. Os dados foram avaliados com ANOVA e teste de Tukey $(p=0,05)$. Resultados: para os tratamentos de consultório, não houve diferença na microdureza entre os tempos de avaliação e entre os agentes clareadores $(p \geq 0,05)$. Já para os tratamentos caseiros, não houve diferença entre os grupos com e sem cálcio, mas, para ambos, ocorreu uma significativa redução na microdureza imediatamente após a conclusão do tratamento, que se manteve após os sete dias de armazenamento em água destilada $(p<0,05)$. Conclusão: o cálcio presente nos agentes clareadores não teve influência na microdureza do esmalte após os tratamentos clareadores caseiro e de consultório.

Palavras-chave: Clareamento dental. Cloreto de cálcio. Dureza.

\section{Introdução}

Ao longo das últimas décadas, há uma busca crescente por melhores condições estéticas e tratamentos clareadores, que se mostram eficazes, seguros e com bons resultados ${ }^{1}$. O clareamento dental é a técnica menos invasiva e mais conservadora, porém pode apresentar algumas desvantagens, como sensibilidade, ulcerações nos tecidos moles e, por vezes, toxicidade ${ }^{2}$. Portanto, o profissional deve ter experiência com o mecanismo de ação e conhecimento da segurança biológica dos agentes clareadores, para poder indicá-los de forma correta e orientar seus pacientes sobre o tratamento. Existem várias técnicas de clareamento, como o clareamento caseiro com moldeira individual, o clareamento em consultório e o clareamento combinado, que associa as técnicas de consultório e caseira. As indicações e os tipos de clareamento estão relacionados às alterações de cor, que podem ser causadas por fatores extrínsecos ou intrínsecos ${ }^{3,4}$, e às características do paciente.

Entretanto, estudos mostram que o peróxido de hidrogênio é um agente oxidante com alta capacidade de penetração no esmalte e na dentina devido ao seu baixo peso molecular ${ }^{2}$ e, dependendo da técnica empregada, da quantidade de sessões, do tempo de exposição e da concentração do agente clareador, pode acarretar efeitos indesejáveis, como a sensibilidade e a alteração da morfologia do esmalte ${ }^{5-9}$. A literatura sugere que o uso indiscriminado dos

\footnotetext{
Cirurgiã-dentista, Universidade de Passo Fundo (UPF), Passo Fundo, Rio Grande do Sul, Brasil.

Cirurgiã-dentista, UPF, Passo Fundo, Rio Grande do Sul, Brasil.

Cirurgiã-dentista, UPF, Passo Fundo, Rio Grande do Sul, Brasil.

Mestre em Odontologia, na área de concentração Clínica Odontológica (UPF). Doutorando no Programa de Pós-Graduação em Odontologia da UPF, Passo Fundo, Rio Grande do Sul, Brasil. Rio Grande do Sul, Brasil.

***** Doutora em Odontologia, na área de concentração Materiais Dentários. Professora assistente do Programa de Pós-Graduação em Odontologia da UPF, Passo Fundo, Rio Grande do Sul, Brasil. Rio Grande do Sul, Brasil.
} 
agentes clareadores poderia resultar em poros, erosões, aumento da rugosidade superficial, redução dos níveis de cálcio e fosfato e diminuição da microdureza do esmalte dental, porém as consequências causadas pelos agentes clareadores ainda não estão totalmente esclarecidas, o que gera contestações ${ }^{10}$.

A utilização do cálcio foi proposta para potencializar a remineralização da superfície do substrato clareado, minimizando a diminuição da microdureza do esmalte ${ }^{11-13}$. Entre as formulações existentes, a mais conhecida contém fosfato e cálcio amorfos e fosfopeptídeo de caseína (casein phosphopeptide-amorphous calcium phosphate - CPP-ACP $)^{14}$, derivado da proteína do leite, usado também para prevenir cáries, apresentando bons resultados após o clareamento e mantendo as propriedades do esmalte $^{1,15,16}$. Esse nanocomplexo atua como um reservatório de cálcio e fosfato, que se prende à placa dental e às superfícies dos dentes. Pode obstruir rapidamente os túbulos dentinários por precipitação rápida de cristais de fosfato e cálcio na superfície e também dentro dos túbulos dentinários, aumentando a remineralização e diminuindo a sensibilida$\mathrm{de}^{17}$. A partir disso, surgiram no mercado inúmeros produtos com o cálcio, que pode estar presente na composição do agente clareador ou ser aplicado em forma de pasta após o procedimento clareador.

Assim, o objetivo deste estudo é avaliar o efeito dos usos de consultório e caseiro dos agentes clareadores com cálcio na microdureza do esmalte dental. A hipótese testada é a de que os dentes tratados com agentes clareadores com cálcio apresentam maior microdureza.

\section{Materiais e método}

Esta pesquisa foi submetida e aprovada pela Comissão de Ética no Uso de Animais da Universidade de Passo Fundo. Oito incisivos bovinos foram limpos com curetas periodontais e tiveram sua coroa separada da raiz com uso de motor de baixa rotação e disco carburundum. As coroas dos incisivos bovinos foram armazenadas em água destilada a $37^{\circ} \mathrm{C}$.

O corte das coroas foi realizado com disco diamantado sob refrigeração constante em uma cortadeira metalográfica (Miniton Struers ${ }^{\mathrm{R}}$, Denmark), e os dentes foram seccionados em quarto fragmentos. Após o corte, um fragmento das coroas foi incluído com resina acrílica autopolimerizável em um anel de PVC. A superfície do esmalte foi planificada e polida em uma politriz (Abramin Struers ${ }^{\mathrm{R}}$, Denmark), com rotação de $250 \mathrm{rpm}$, usando lixas d'agua de granulações 800 e 1.200, sob refrigeração constante. Os dentes foram armazenados em água destilada até o início do experimento.

Cada fragmento de uma mesma coroa recebeu um tipo de tratamento clareador (Tabela 1) $(n=8)$ : (Con) protocolo de consultório com peróxido de hidrogênio 35\% (HP 35\%); (Con+Ca) protocolo de consultório com HP 35\% com cálcio; (Cas) protocolo caseiro com peróxido de carbamida $10 \%$; (Cas+Ca) protocolo caseiro com HP 6\% com cálcio.

Tabela 1 - Grupos experimentais

\begin{tabular}{|c|c|c|c|c|c|}
\hline Grupo & Agente & Concentração & Marca & Protocolo & Cálcio \\
\hline $\mathrm{Con}+\mathrm{Ca}$ & $\begin{array}{l}\text { Peróxido de } \\
\text { hidrogênio }\end{array}$ & $35 \%$ & $\begin{array}{l}\text { Whitness HP Blue } \\
\text { Calcium }^{R} \text { (FGM) }\end{array}$ & Consultório: aplicação única (40 min) & Sim \\
\hline Con & $\begin{array}{l}\text { Peróxido de } \\
\text { hidrogênio }\end{array}$ & $35 \%$ & $\begin{array}{l}\text { Whitness HP Maxx } \\
\text { (FGM) }\end{array}$ & Consultório: 2 aplicações (15 min) & Não \\
\hline Cas+Ca & $\begin{array}{l}\text { Peróxido de } \\
\text { hidrogênio }\end{array}$ & $6 \%$ & $\begin{array}{l}\text { White } \text { Class }^{\mathrm{R}} \text { com cálcio } \\
\text { (FGM) }\end{array}$ & $\begin{array}{l}\text { Caseiro: } 1 \text { aplicação/dia durante } \\
\text { 1h } 30 \text { min por } 14 \text { dias }\end{array}$ & Sim \\
\hline Cas & $\begin{array}{l}\text { Peróxido de } \\
\text { carbamida }\end{array}$ & $10 \%$ & Whitness Perfect ${ }^{R}$ (FGM) & $\begin{array}{c}\text { Caseiro: } 1 \text { aplicação/dia durante } \\
1 \mathrm{~h} 30 \mathrm{~min} \text { por } 14 \text { dias }\end{array}$ & Não \\
\hline
\end{tabular}

Fonte: elaboração dos autores.

Os géis clareadores foram aplicados na superfície vestibular dos dentes, na espessura de uma película de aproximadamente $1 \mathrm{~mm}$, com o auxílio de microbrush. Os agentes foram aplicados de acordo com o protocolo preconizado pelo fabricante (Tabela 1). Após cada sessão de clareamento, o gel foi removido com gaze e os corpos de prova foram lavados com água por 10 segundos. Os corpos de prova foram armazenados em água destilada a $37^{\circ} \mathrm{C}$, durante os tratamentos e no período de sete dias entre o fim do tratamento e a última avaliação de microdureza.

Os testes de microdureza foram realizados com microdurômetro (Shimadzu ${ }^{R}$ HMV-G 20S, São Pau- lo, SP, Brasil). Os dentes foram posicionados com a superfície do esmalte perpendicular ao longo eixo de um edentador Vickers, sendo aplicada uma carga de 200 g por 15 segundos. Foram realizadas três medidas de edentações por corpo de prova. A microdureza foi avaliada antes (T0), imediatamente após as aplicações (T1) e sete dias após a conclusão dos tratamentos (T2). No caso dos tratamentos de consultório, as medidas T0 e T1 foram feitas no mesmo dia. Para os tratamentos caseiros, a medida T1 foi feita após 14 dias de tratamento caseiro.

Os valores de microdureza foram analisados estatisticamente com ANOVA pareado e teste de Tukey, com nível de significância de 5\%. 


\section{Resultados}

Para os tratamentos de consultório, não houve diferença na microdureza entre os tempos de avaliação e entre os agentes clareadores $(\mathrm{p} \geq 0,05)$ (Tabela 2).

Tabela 2 - Valores de média (desvio padrão) de microdureza (HV) para os tratamentos clareadores de consultório nos diferentes tempos ${ }^{\text {ns }}$

\begin{tabular}{l|c|c|c}
\cline { 2 - 4 } \multicolumn{1}{c|}{} & T0 & T1 & T2 \\
\hline Con & $324(9)$ & $313(8)$ & $323(23)$ \\
Con+Ca & $323(10)$ & $322(9)$ & $312(39)$ \\
\hline
\end{tabular}

Fonte: elaboração dos autores.

*ns - sem significância estatística.

Para os tratamentos caseiros, houve diferença na microdureza entre os tempos de avaliação $(\mathrm{p}<0,05)$. Houve uma redução significativa da microdureza imediatamente após a conclusão dos tratamentos caseiros, que se manteve após sete dias de armazenamento em água destilada. Não houve diferença significativa na microdureza entre os agentes clareadores com e sem cálcio nos diferentes tempos avaliados ( $\mathrm{p} \geq 0,05)$ (Tabela 3 ).

Tabela 3 - Valores de média (desvio padrão) de microdureza (HV) para os tratamentos clareadores caseiros nos diferentes tempos

\begin{tabular}{|c|c|c|c|}
\hline & T0 & T1 & T2 \\
\hline Cas & 347 (24) a A & 302 (11) b A & 314 (31) b A \\
\hline $\mathrm{Cas}+\mathrm{Ca}$ & $341(39)$ a A & 292 (50) b A & 299 (47) b A \\
\hline
\end{tabular}

Fonte: elaboração dos autores.

* Médias seguidas por letras minúsculas iguais na mesma linha não têm diferença estatística ( $p \geq 0,05)$.

** Médias seguidas por letras maiúsculas iguais na mesma coluna não têm diferença estatística $(p \geq 0,05)$.

\section{Discussão}

Existe uma grande variedade de produtos e métodos para o clareamento de dentes vitais, com diferentes tipos e concentrações de agentes clareadores, tempos de aplicações e modos de aplicações ${ }^{18}$. Assim, muitos estudos investigam os prováveis danos que os agentes clareadores podem causar na estrutura dental, mesmo sendo este o tratamento mais conservador em comparação com restaurações de resina e facetas de cerâmica ${ }^{19}$.

Com os objetivos de reduzir possíveis efeitos deletérios no esmalte dental e aumentar a resistência à desmineralização do esmalte, surgiram agentes clareadores modificados com adição de cálcio ${ }^{10}$. A adição dos íons cálcio ao gel seria capaz de promover a saturação do agente clareador e diminuir a perda de minerais do esmalte durante o tratamento, aumentando, consequentemente, a resistência à desmineralização ${ }^{20}$. Além disso, Ca adicionado a soluções ácidas é capaz de diminuir a perda mineral do esmalte em até $50 \%{ }^{21,22}$.
Porém, no presente estudo, observou-se que a presença de cálcio não afetou a microdureza do esmalte dental imediatamente (T1) e após sete dias da conclusão do tratamento clareador (T2), rejeitando a hipótese do estudo. A maior parte dos estudos concorda que as presenças de Ca e F seriam capazes de reduzir os efeitos deletérios dos agentes clareadores ${ }^{10,23}$. Alguns estudos observaram diminuição da microdureza do esmalte após o clareamento, mas relataram que essa redução foi minimizada nas amostras que receberam tratamento com os agentes clareadores que continham adição de cálcio ${ }^{10}$.

A diferença de resultados encontrados entre os estudos pode estar relacionada ao tipo de metodologia escolhida para realizar o experimento, como o meio de armazenagem das amostras antes e após receber o clareamento. No presente estudo, foi utilizada água destilada, mas, em outras pesquisas, já foram utilizadas solução remineralizante, saliva artificial e saliva humana ${ }^{12,24}$. O meio de armazenagem é muito importante, porque alguns estudos verificaram que a saliva é um contribuinte essencial na recuperação do conteúdo mineral ${ }^{11,25-27}$.

Ainda, no estudo de Cavalli et al. ${ }^{12}$ (2010), observou-se que o uso de uma solução remineralizante contribuiu para a reparação de defeitos microestruturais promovidos por ação de agentes por meio de adsorção e precipitação de componentes salivares, tais como Ca e fosfato. No presente estudo, optou-se por usar água destilada para verificar o efeito da adição de cálcio no agente clareador sem a presença de fatores confundidores, como a possível influência da saliva artificial.

Além disso, o tipo de cálcio na composição do agente clareador testado (gluconato de cálcio) não é o mesmo CPP-ACP encontrado na maioria dos estudos $^{28,29}$. Em um estudo que usou CPP-ACP diluído no agente clareador, foi encontrada diminuição na microdureza do esmalte ${ }^{30}$. Esses resultados comprovam que agentes clareadores contendo cálcio podem não ter o mesmo potencial remineralizador do que a pasta de CPP-ACP utilizada após cada sessão clareadora. De Abreu et al. ${ }^{11}$ (2011) avaliaram o efeito da adição de fosfato de cálcio amorfo (ACP) aos agentes clareadores, concluindo que apenas os agentes de peróxido de hidrogênio de baixa concentração se beneficiaram da presença desse componente.

Segundo os fabricantes, uma vantagem dos géis clareadores contendo cálcio seria a capacidade de manter o pH alto $(8-9)$ e estável durante o clareamento de consultório, ao contrário do que ocorre com géis que não possuem cálcio em sua formulação. Os produtos clareadores deveriam apresentar um $\mathrm{pH}$ relativamente neutro para minimizar os efeitos deletérios do clareamento ${ }^{17}$. Os agentes utilizados na presente pesquisa apresentam $\mathrm{pH}$ na faixa de $6-7$, que é considerado neutro. Isso poderia explicar a estabilidade nos valores de microdureza após o clareamento com os agentes clareadores de 
consultório. Entretanto, análises químicas adicionais para verificar a estabilidade do $\mathrm{pH}$ durante $o$ clareamento e a quantidade de cálcio são necessárias para a melhor compreensão dos mecanismos de ação desses agentes.

Por sua vez, os agentes clareadores caseiros diminuíram a microdureza do esmalte após o tratamento. Dois tipos de agentes foram avaliados no tratamento caseiro, peróxido de carbamida $10 \%$ e peróxido de hidrogênio 6\%, e ambos reduziram a microdureza do esmalte dental. Isso está diretamente relacionado ao tempo de contato com o agente clareador $^{6-9}$. Apesar de esses agentes apresentarem menor concentração do que os agentes de consultório, eles permaneceram em contato com o esmalte por mais tempo. Clinicamente, esse efeito é reduzido pelo efeito da presença de saliva humana, que pode eliminar o efeito desmineralizante causado pelo baixo $\mathrm{pH}$ dos agentes. Portanto, as alterações de superfície do esmalte observadas nos estudos in vitro podem não ocorrer de forma tão agressiva in $v i v o^{13}$. A utilização constante de fluoretos, a adoção de medidas adequadas de higiene oral e a presença da saliva poderiam aumentar a dureza do esmalte durante e depois do clareamento.

\section{Conclusão}

O cálcio presente nos agentes clareadores não teve influência na microdureza do esmalte após os tratamentos clareadores caseiros e de consultório.

\section{Agradecimentos}

Os autores agradecem a doação dos agentes clareadores pela empresa FGM Produtos Odontológi$\cos$ (Joinville, SC, Brasil).

\section{Abstract}

Objective: to evaluate the effect of bleaching agents with calcium in the dental enamel microhardness. Materials e method: the study is experimental, in vitro and with bovine teeth. Eight crowns of bovine incisors were sectioned into 4 parts and divided into 4 groups, according to the type of bleaching agent $(n=8)$ : (Con) in-office protocol (35\% hydrogen peroxide - HP $35 \%) ;(\mathrm{Com}+\mathrm{Ca})$ in-office protocol with calcium (HP 35\%); (Cas) at-home protocol (10\% carbamide peroxide); (Cas+Ca) at-home protocol with calcium (HP 6\%). Samples were treated according to the manufacturer's recommendations for each bleaching agent. The enamel microhardness was evaluated before, immediately after and 7 days after the bleaching treatments. A microdurometer and Vickers tip were used, with a load of $200 \mathrm{~g}$ for $15 \mathrm{~s}$. Samples were stored in distilled water at $37^{\circ} \mathrm{C}$ during the experiment. Data were analyzed with ANOVA and Tukey's test ( $p=0.05$ ). Results: for in-office treatments, there was no difference in the microhardness for the evaluated periods and among the bleaching agents ( $p \geq 0.05)$. However, for the at-home treatments, there was no difference between the groups with and without calcium, but for both agents a significant reduction in microhardness occurred immediately after the conclusion of the treatment, and was maintained after 7 days of storage in distilled water $(p<0.05)$. Conclusion: the calcium present in the bleaching agents had no influence on the microhardness of the enamel after the at-home and in-office bleaching treatments.

Keywords: Tooth bleaching. Calcium chloride. Hardness.

\section{Referências}

1. Borges BC, Borges JS, De Melo CD, Pinheiro IV, Santos AJ, Braz R, et al. Efficacy of a novel at-home bleaching technique with carbamide peroxides modified by cpp-acp and its effect on the microhardness of bleached enamel. Oper Dent 2011; 36(5):521-8.

2. Dillenburg ALK, Conceição EN. Clareamento dental. Dentística, Saúde e Estética Artmed 2007; 2:236-63.

3. Bizhang M, Chun Y-HP, Damerau K, Singh P, Raab WH-M, Zimmer S. Comparative clinical study of the effectiveness of three different bleaching methods. Oper Dent 2009; 34(6):635-41.

4. Bernardon JK, Sartori N, Ballarin A, Perdigão J, Lopes G, Baratieri LN. Clinical performance of vital bleaching techniques. Oper Dent 2010; 35(1):3-10.

5. Soares FF, Sousa JAC, Maia CC, Fontes CC, Fontes CM, Cunha LG, et al. Clareamento em dentes vitais: uma revisão de literatura. Rev Saúde.Com 2008; 4:72-84.

6. Abouassi T, Wolkewitz M, Hahn P. Effect of carbamide peroxide and hydrogen peroxide on enamel surface: an in vitro study. Clin Oral Invest 2011; 15(5):673-80.

7. Metz MJ, Cochran MA, Matis BA, Gonzalez C, Platt JA, Lund MR. Clinical evaluation of $15 \%$ carbamide peroxide on the surface microhardness and shear bond strength of human enamel. Oper Dent 2007; 32(5):427:36.

8. Faraoni-Romano JJ, Silveira AG, Turssi CP, Serra MC. Bleaching agente with varying concentrations of carbamide and/ or hydrogen peroxides: effect on dental microhardness and roughness. J Esthet Restor Dent 2008; 20(6):395-402.

9. Coceska E, Gjorgievska E, Coleman NJ, Gabric D, Slipper IJ, Stevanovic M, et al. Enamel alteration following tooth bleaching and remineralization. J Microsc 2016; 262(3):232-44.

10. Amaral PG, Sales GCF, Santos LR, Magalhães DBL, Gusmão ES. Influência da presença do cálcio em agentes clareadores e sua relação com a microdureza do esmalte dental humano. Rev Bras Ciências da Sáude 2010; 14:37-42.

11. De Abreu RD, Sasaki TR, Amaral BLF, Flório M, Basting TR. Effect of home-use and in-office bleaching agents containing hydrogen peroxide associated with amorphous calcium phosphate on enamel microhardness and surface roughness. J Esth Rest Dent 2011; 23(3):158-68.

12. Cavalli V, Rodrigues AKL, Paes-Leme FA, Brancalion LM, Arruda ZAM, Berger BS, et al. Effects of bleaching agents containing fluoride and calcium on human enamel. Quintessence Int 2010; 41(8):157-65.

13. Lago AD, Oliveira FS, Freitas PM, Ferreira SS, Netto NG. Avaliação da microdureza do esmalte após clareamento com peroxido de hidrogênio a $35 \%$ contendo cálcio. Rev Pesq Saúde 2014; 15:285-9. 
14. Reynolds EC. Casein phosphopeptide-amorphous calcium phospate: the scientific evidence. Adv Dent Res 2009; 21(1):25-9.

15. Bayrak S, Tunc ES, Sonmez IS, Egimez T, Ozmen B. Effects of casein phosphopeptide-amorphous calcium phosphate (CPP-ACP) application on enamel microhardness after bleaching. Am J Dent 2009; 22(6):393-6.

16. Cunha AGG, Vasconcelos AAM, Borges BCD, Vitoriano JO, Alves-Junior C, Machado CT, et al. Efficacy of in-office bleaching techniques combined with the application of a casein phosphopeptide-amorphous calcium phosphate paste at different moments and its influence on enamel surface properties. Microsc Res Tech 2012; 75(8):1019-25.

17. Vasconcelos AAM, Cunha AGC, Borges BCD, Machado CT, Santos AJS. Tooth whitening with hydrogen/carbamide peroxides in association with a CPP-ACP paste at different proportions. Aust Dent J 2012; 57(2):213-9.

18. Polydorou O, Wirsching M, Wokewitz M, Hahn P. Clinical three-month evaluation of vital tooth bleaching using light units - a randomized clinical study. Oper Dent 2013; $38(1): 21-32$

19. Yesiilyurt C, Sezer U, Ayar MK, Alp CK, Tasdemir T. The effect of a new calcium-based agent, Pro-Argin, on the microhardness of bleached enamel surface. Aust Dental J 2013; 58(2):207-12.

20. Giannini M, Silva AP, Cavalli V, Paes Leme AF. Effect of carbamide peroxide-based bleaching agents containing fluoride or calcium on tensile strength of human enamel. J Appl Oral Sci 2006; 14(2):82-7.

21. Perdigão J, Franci C, Swift EJ Jr, Ambrose WW, Lopes M. Ultra-morphological study of the interaction of dental adhesives with carbamide peroxide- bleached enamel. Am J Dent 1998; 11(6):291-301

22. Hughes JA, West NX, Parker DM, van der Braak MH, Addy M. Effects of $\mathrm{pH}$ and concentration of citric; malic and lactic acids on enamel; in vitro. J Dent 2000; 28(2):147-52.

23. Cavalli V, Rodrigues LKA, Paes-Leme AF, Soares LES, Martini AA, Berger SB, et al. Effects of the addition of fluoride and calcium to low-concentrated carbamide peroxide agents on the enamel surface and subsurface. Photomed Laser Surg $2011 ; 29(5): 319-25$

24. Kim YS, Kwon HK, Kim BI. Effect of nano-carbonate apatite to prevent re-stain after dental bleaching in vitro. J Dent 2011; 39(9):636-42.

25. Zanet CG, Fava M, Alves LA. In vitro evaluation of the microhardness of bovine enamel exposed to acid solutions after bleaching. Braz Oral Res 2011; 25(6):562-7.

26. Sa Y, Sun L, Wang Z, Ma X, Liang S, Xing W, et al. Effects of two in office bleaching agents with different ph on the structure of human enamel: an in situ and in vitro study. Oper Dent 2013; 38(1):100:10.

27. Sa Y, Wang Z, Ma X, Lei C, Liang S, Sun L, et al. Investigation of three home applied bleaching agents onenamel structure and mechanical properties: an in situ study. J Biomed Opt 2012; 17(3):1-9.

28. Oliveira R, Paes Leme AF, Giannini M. Effect of a carbamide peroxide bleaching gel containing calcium or fluoride on human enamel surface microhardness. Braz Dent J 2005; 16(2):103-6

29. Oshiro M, Yamaguchi K, Takamizawa T, Inage H, Watanabe T, Irokawa A, et al. Effect of CPP-ACP paste on tooth mineralization: an FE-SEM study. J Oral Sci 2007; 49(2):115-20.
30. Sasaki RT, Catelan A, Bertoldo CES, Venâncio PC, Groppo FC, Ambrosano GMB, et al. Effect of 7,5\% hydrogen peroxide containing remineralizing agentes on hardness, color change, roughness and micromorphology of human enamel. Am J Dent 2015; 28(5):261-7.

\section{Endereço para correspondência:}

Márcia Borba

Faculdade de Odontologia

Universidade de Passo Fundo

Campus 1, BR 285, Km 171, Bairro São José

CEP 99052-900 - Passo Fundo, RS

Telefone: (55) 54-3316-8395

E-mail: marciaborba@upf.br marcia_borb@hotmail.com

Recebido: 23/09/17. Aceito: 20/11/17. 